\title{
LINGUISTIQUE
}

\author{
BRUNON BULIKOWSKI
}

Université Adam Mickiewicz à Poznań

\section{L'OPPOSITION ENTRE NOM ET VERBE : LE PROBLÈME DE L'INFINITIF}

\begin{abstract}
A bstract. Bulikowski Brunon, L'opposition entre nom et verbe : le problème de l'infinitif [Opposition between the noun and the verb: problem of the infinitive]. Studia Romanica Posnaniensia, Adam Mickiewicz University Press, Poznań, vol. XXXII : 2005, pp. 3-12. ISBN 83-232-1465-4, ISSN 01372475 .

In this article the author considers grammatical properties of the infinitive in the French language, Because the French infinitive possesses properties both typical of the verb and of the noun, in order to define its grammatical status it is necessary to mark the boundaries between the two mentioned categories. To illustrate the complexity of this task the author refers to languages in which verbalnominal opposition differs from that in the French language. Both morphological and syntactic aspects have heen here taken into consideration. In the final part of the article the author quotes an interesting theory of G. Guillaume which allows the classification of grammatical categories, the infinitive included, on the basis of the relation of semantic character occurring between them.
\end{abstract}

L'opposition verbo-nominale est un problème dont on ne saurait parler sans prendre en compte les différents aspects morpho-syntaxiques de l'emploi de l'infinitif. Les hésitations concernant le vrai statut grammatical de l'infinitif décrites par les linguistes francophones semblent réapparaître de façon spectaculaire lors de la traduction de cette catégorie en polonais. Souvent, le traducteur n'utilise pas, dans ce cas, le verbe équivalent, mais il fait appel à un nom lié dérivationnellement avec ce demier. Regardons quelques exemples d'infinitif français à fonction sujet de la phrase :

(A) Chasser ne m'attirait nullement.

Polowanie zupetnie mnie nie pociagalo.

(B) Fumer nuit à la santé.

Palenie szkodzi zdrowiu. 
(C) Et maintenant elle savait que vivre ne vaut pas tant d'inquiétude (A. France). A teraz wiedziata, że życie nie jest warte tyle niepokoju.

Dans les trois phrases, l'infinitif sujet sera rendu à l'aide d'un substantif verbal (Sv), respectivement polowanie, palenie, zycie. Il faut pourtant remarquer que ce type de changement ne concerne pas tous les verbes et que, suivant le lexème, on peut s'attendre à la traduction à l'aide aussi bien d'un nom que d'un infinitif (Inf) :

Former les jeunes est une tâche difficile.

Ksztatcenic mlodych jest trudnym zadanien. (Sv)

Ksziatcić mlodych jest trudnym zadaniem. (Inf)

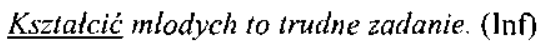

Enfin, dans le cas de certaines phrases, l'infinitif ne pourra être rendu en por lonais que par une forme personnelle du verbe :

Avoir le métro à proximité leur a changé la vie.

To, ze maja metro w poblizu, zmienilo ich życie.

* Mieć metro w poblizu zmienilo ich życie. (Inf)

Quoi qu'il en soit, le fait qu'on recours fréquemment à un nom d'action lors de ce type de traductions incite certainement à la réflexion sur le concept de catégorie grammaticale lui-même et plus spécialement sur les frontières entre différentes catégories ainsi que les critères qui permettent de les fixer.

Les changements décrits ci-dessus rappellent la notion de métataxe, qui est une application de l'indépendance du structural et du sémantique. Le simple appel à une catégorie grammaticale différente dans l'autre langue est la forme la plus simple de ce processus (Tesnière $1965: 284$ ). Nous pouvons donc parler de métataxe de l'infinitif en nom dans les exemples $\mathrm{A}, \mathrm{B}$ et $\mathrm{C}-$ toutefois - à condition d'admettre que les mots chasser, fumer, voir dans les phrases étudiées soit des verbes. Car il s'avère que le statut de l'infinitif, considéré généralement comme une catégorie verbale, est fort discutable, et que certaines de ses propriétés le font rapprocher du nom. Avant de passer à la description détaillée de ces propriétés, il y a lieu d'aborder le problème des critères qui permettent d'établir les limites entre le nom et le verbe, quel que soit le mode de ce dernier.

En premier lieu, prenons en considération le critère sémantique. Dans de nombreux manuels de grammaire et dictionnaires, la définition du verbe comporte l'indication selon laquelle c'est l'élément qui exprime une action ou un état, et qui situe le procès dans le temps.

VERBE - Mot qui exprime une action, un état, un devenir, et qui présente un système complexe de formes, notamment dans les langues indo-européennes (Nouveau Petit Robert 1993).

Il est pourtant facile de remarquer que d'autres catégories, comme le nom et l'adjectif peuvent aussi exprimer une action ou un état (digestion, attentif, etc.). De 
l'autre côté, des notions comme l'antériorité, la postériorité ou la successivité temporelle peuvent s'exprimer aussi bien par un verbe qu'au moyen d'un préfixe (une présupposition, un ex-président), d'un adjectif (ma future fiancée), d'une préposition (après, pendant) ou d'un adverbe (demain, successivement). Il en résulte que la propriété d'exprimer un procès ou de le situer dans le temps n'est pas réservée uniquement à la classe de verbe et que, pour donner une définition plus satisfaisante du verbe, il est nécessaire de prendre en considération d'autres facteurs. Citons ici André Martinet : «En français, pleuvoir est un verbe, non point parce qu'il exprime un procès, mais bien parce qu'il se fléchit sur un modèle qui est celui d'une foule de mots qui, traditionnellement, ont reçu la dénomination de verbes; pluie est un nom, non parce qu'il désigne une chose, mais parce qu'il entre dans certains types de combinaisons qui caractérisent les complexes appelés „noms”, par opposition à d'autres complexes qu'on désigne sous d'autres termes » (Martinet 1965 : 197).

L'étude de différentes langues montre que la classification des catégories grammaticales doit être envisagée tant sur le plan de la morphologie que celui de la syntaxe. Dans le cadre de cette demière, il est utile de distinguer, d'un côté, la syntaxe du syntagme (syntaxe "immédiate ") qui étudie les combinaisons du mot avec ses satellites; et de l'autre côté, la syntaxe de proposition (syntaxe phrastique), qui décrit la nature et les rapports du prédicat. Il y a lieu de préciser ici que dans notre étude nous adoptons la notion de prédicat qui correspond à l'un des deux éléments constitutifs de l'énoncé minimum.

Dans beaucoup de langues, la distinction entre le verbe et le nom s'étend aussi bien sur la morphologie que sur la syntaxe immédiate et la syntaxe phrastique. Ainsi, on voit que tant en français qu'en polonais la morphologie nominale, variation en nombre, en genre, en cas (en polonais), s'oppose nettement à la morphologie verbale (conjugaison). De même, la syntaxe immédiate du nom et celle du verbe n'ont rien de commun : article, épithète avec accord en genre et nombre, "complément de nom », etc., d'un côté, adverbe, objet direct, etc., de l'autre.

Quant à la syntaxe phrastique, le verbe significatif (à la forme personnelle) est toujours prédicat; le nom est actant. De plus, même dans les phrases où l'on dit habituellement qu'un nom est prédicat (ou, selon la grammaire traditionnelle, attribut), la copule verbale est normalement nécessaire. Elle n'est omise que dans des énoncés particuliers, marqués, comme :

Absurde, cette idée.

Dans les phrases neutres, ordinaires du type

Pierre est avocat,

elle ne saurait manquer. Si dans ces phrases le nom est le centre sémantique du groupe prédicatif (ici: est avocat), le verbe en est le centre grammatical (Lazard 1984 : 32). 
Bien qu'elle traverse toute la structure morphosyntaxique la distinction entre nom et verbe dans les deux langues ne s'impose pas de manière rigoureuse aux racines. Ainsi, par exemple, nous rencontrons les mêmes racines tant dans le nom course et dans le verbe courser qui en dérive, que dans les mots bouffer et bouffe où diachroniquement la dérivation s'est faite en sens inverse. Il existe aussi de nombreux exemples de ce type en polonais, par exemple : krzyż - krzyżować ou biegać-bieg.

L'opposition susdite n'est done pas aussi forte en morphologie que, par exemple, en esquimau où les racines forment deux classes disjointes (sauf quelques exceptions) : les unes admettent les suffixes nominaux et les désinences casuelles, les autres les suffixes verbaux et les désinences de mode et de personne. De plus, le nom n'est jamais prédicat dans cette langue. Si l'on veut faire d'une notion nominale le prédicat logique d'une relation, il faut de ce nom dériver un verbe au moyen du suffixe approprié (ibid. : 32-33).

Contrairement à l'esquimau, dans certaines langues océaniennes, la distinction entre nom et verbe repose essentiellement sur la syntaxe immédiate. C'est le cas, par exemple, du tongan, langue parlée dans les îles Tonga, où l'on atteste de nombreux lexèmes qui peuvent fonctionner aussi bien comme noms que comme verbes suivant qu'ils se combinent avec les particules nominales, notamment des articles antéposés au lexème, ou avec les particules verbales, comme des particules exprimant l'aspect et le temps, et des pronoms sujets antéposés. Les formes nominales remplissent les fonctions actancielles, mais, d'autre part, tant les formes verbales que les formes nominales peuvent fonctionner comme prédicat. Dans ce dernier cas, la phrase commence par une particule spécifique $(k o)$ suivie du prédicat nominal (ibid. : 34-35). Ainsi dans les phrases

$$
\begin{array}{ll}
\eta_{\text {oku }} \eta_{i} \eta_{a} \text { Nuku } & \text { "Nuku est roi " } \\
\text { ko e } t u \eta_{i} \eta_{a} \text { Nuku } & \text { «Nuku est roi» }
\end{array}
$$

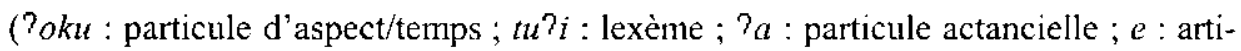
cle) les séquences $7 o k u t u ? i$ et $e t u ?_{i}$ correspondent respectivement à une forme verbale et une forme nominale.

Il paraît pourtant que la distinction entre nom et verbe est la plus difficile à saisir dans les langues indonésiennes. En malgache par exemple, elle repose toute entière sur la morphologie. Les formes verbales qui ne comportent aucune conjugaison personnelle, peuvent, tout comme les formes "nominales ", être précédées d'un article :

malalao ny ankizy « les enfants jouent »

ankizy ny malalao " ceux qui jouent sont les enfants"

(malalao « jouer », ankizy « enfant », ny article)

De plus, tant les formes verbales que nominales sont liées par les mêmes procédés à un déterminant postposé, qui est agent dans le cas d'un verbe, et possesseur dans celui d'un nom : 
$\begin{array}{ll}\text { trano-ko } & \text { "maison à moi » } \\ \text { tia-ko } & \text { "aimé par moi } ~\end{array}$

(trano « maison », tia « aimer », -ko suffixe pronominal)

Beaucoup d'affixes sont aussi bien "nominaux » que "verbaux »: c'est le cas des affixes de temps. Le trait le plus net qui permet de distinguer des noms et des verbes est la capacité qu'ont ces derniers de varier en voix. Un lexème qui n'admet aucune variation en voix, même s'il désigne une action ou un état, ne se distingue plus d'une forme nominale (ibid. : 36-37).

La présentation ci-dessus montre que la différenciation entre nom et verbe se manifeste, selon les langues, inégalement dans les trois zones de la structure morpho-syntaxique. Elle peut être essentiellement morphologique ou syntaxique. Elle peut concerner toutes les trois zones ou être limitée à deux ou à une seule.

L'infinitif se présente comme une espèce intermédiaire entre la catégorie du verbe et celle du substantif. C'est une catégorie mixte qui peut avoir un nombre plus ou moins grand de caractères verbaux ou substantivaux. Il semble que sa forme la plus proche du substantif est celle dont on se sert pour désigner un verbe donné comme notion (Tesnière, op.cit. : 418-419), par exemple dans la phrase

Conjuguez le verbe aimer!

Toutefois, il faut souligner que cette façon de désigner le verbe par l'infinitif n'est pas universelle. Dans les langues sémitiques, par exemple, les grammairiens désignent le verbe par la troisième personne du singulier masculin du parfait de la forme simple :

(arabc) le verbe quatala : le verbe "il a tué »

L'usage de désigner le verbe par l'infinitif n'est même pas toujours possible. Dans certaines langues, l'infinitif n'existe pas, ou du moins y est d'usage fort restreint. C'est le cas en bulgare. Les grammairiens désignent le verbe, dans cette langue, par la première personne du présent de l'indicatif :

le verbe obicam : le verbe « $j$ ' aime »

Parmi les caractéristiques essentielles du verbe qui se maintiennent le plus longtemps à l'infinitif est la catégorie grammaticale de la voix. Aussi la plupart des langues qui connaissent l'infinitif distinguent-elles l'infinitif actif et l'infinitif passif.

\begin{tabular}{ll}
\multicolumn{1}{c}{ actif } & \multicolumn{1}{c}{ passif } \\
fr. aimer & être aimé \\
ang. to love & to be loved \\
pl. kochać & byc kochanym
\end{tabular}


Il existe de même un infinitif réfléchi et un infinitif réciproque :

Se regarder dans un miroir. (i. réfléchi)

Il faut s'entraider. (i. réciproque)

Toutefois, la notion de voix est loin d'être aussi nette dans l'infinitif que dans le verbe. En français, la voix de l'infinitif est parfois ambiguë (Tesnière, op. cit. : 427) :

J'ai vu manger des chiens.

Widzialem, jak psy jedza. (des chiens mangent)

Widzialem, jak jedza psy. (des chiens sont mangés)

À la différence de la catégorie de la voix, la catégorie de la personne est une des catégories verbales qui disparaît le plus tôt et le plus généralement de l'infinitif. Tant en français qu'en polonais, l'infinitif n'est pas susceptible d'exprimer la personne. C'est pourquoi on le range parmi les modes impersonnels.

Du verbe dont il dérive, l'infinitif conserve la faculté d'être le régissant de deux types de subordonnés, les actants

Réussir une mayonnaise est chose délicate

et les circonstants

Il espère réussir facilement.

Inversement, du nom, il acquiert la faculté d'assumer le rôle d'actant. C'est par exemple le cas de la phrase

Réussir encourage à persévérer.

La preuve, c'est que, si on remplace, dans cet exemple, l'infinitif par un vrai substantif de sens équivalent tel que la réussite ou le succès, celui-ci est également premier actant du verbe:

La réussite encourage à persévérer.

Le stuccès encourage à persévérer.

Ici il est intéressant d'examiner de plus près le cas de propositions dites infinitives comme

J'ai entendu Pierre chanter.

Selon Gustave Guillaume, la fonction de second actant est remplie dans cette construction non seulement par le nom Pierre, mais aussi par l'infinitif. Pierre qui est second actant de $j$ 'ai entendu ne peut assumer en même temps la fonction de prime actant. D'après Guillaume, un substantif dans le discours ne peut avoir plus d'une seule fonction. Dans l'exemple ci-dessus, la fonction de chanter n'est pas celle de prédicat, car il faudrait pour cela que Pierre soit en fonction de prime actant. Comme il n'est pas prédicat par rapport à Pierre, l'infinitif chanter ne peut avoir 
d'autre fonction que de continuer la fonction de second actant, dont Pierre est détenteur. De sorte que la véritable analyse est la suivante :

J'ai entendu / Pierre (objet /)/ chanter (objet 2)

Il s'agit donc d'un groupe formant un second actant complexe, déterminé pour partie dans le plan nominal, sous la forme du mot Pierre, et dans le plan verbal, sous la forme de l'infinitif chanter. Entre Pierre et chanter, il n'y a donc pas changement de fonction - la fonction reste la même, celle de second actant - mais changement du plan de détermination de la fonction considérée: le plan pour Pierre est le plan nominal et pour chanter le plan verbal (Guillaume 1987 : 31-33).

Du point de vue logique, Pierre est incontestablement le sujet de chanter, mais d'après Guillaume, la langue repousse cette relation logique. Considérer l'infinitif, dans ce type de constructions comme un prime actant équivaut selon lui à mêler la grammaire et la logique.

Il existe un type de phrases où la nature ambigüe de l'infinitif est soulignée par l'emploi de la copule est qui établit entre l'infinitif sujet et le substantif attribut une sorte d'égalité morphosyntaxique. C'est ainsi que dans

Chanter, c'est ma façon de me battre et de croire (Rostand),

l'infinitif chanter est sur le même plan morphosyntaxique que façon qui est un authentique substantif.

Au fur et à mesure que le verbe devient substantif, parallèlement l'adverbe qui lui est subordonné a tendance à être remplacé par un adjectif. Cette substitution est le signe que l'infinitif a totalement perdu sa nature verbale et complètement acquis sa nature substantivale :

le petit déjeuner

Pourtant, on observe ici des faits de retard. C'est ainsi que le subordonné reste parfois adverbe, malgré l'apparition de la caractéristique substantivale sous la forme de l'article devant l'infinitif :

au pis aller

L'infinitif est susceptible de prendre la marque du pluriel dès qu'il n'est plus senti comme verbe, mais entièrement comme substantif (Tesnière, op. cit. : 436). L'emploi de l'article est alors obligatoire dans les mêmes conditions syntaxiques que pour le substantif :

les dires d'un expert

La nature entièrement substantivale de l'infinitif précédé d'un article ressort aussi de la possibilité de le joncter avec un véritable substantif (ibid. : 437) : 
Dans la théorie de Gustave Guillaume, le statut grammatical de l'infinitif est analysé sur le plan de l'opposition entre l'univers-espace et l'univers-temps. Le nom et le verbe sont ici deux formes aperturales du système de toutes les catégories grammaticales prédicatives.

Selon Guillaume, la construction de la langue est basée sur deux types d'opérations qui altement continuellement : le mouvement qui va de l'universel au singulier et celui qui va du singulier à l'universel. La première opération livre la matière notionnelle du mot, c'est une opération de discernement : « il s'agit de distinguer au sein d'un ensemble contemplé une chose particulière contenue sur laquelle s'arrête l'esprit et qu'il isole de toute autre, afin de la considérer séparément " (Guillaume 1973 : 87). L'autre opération, dite opération d'entendement, permet de comprendre la matière notionnelle sous la forme la plus générale possible, celle de catégorie grammaticale. Elle consiste à « reverser dans l'universel, aux fins d'intellection généralisatrice, le particulier qu'on en a abstrait et qui, en conséquence, ne s'y trouve plus - de sorte que l'universel contemplé, plein au premier battement, apparaît vide au troisième et ne peut plus, dès lors, être particularisé qu'au titre de sa forme » (ibid. : 87-88).

A l'univers-espace et à l'univers-temps susmentionnés, dans la théorie de Guillaume, correspondent respectivement le plan du nom et le plan du verbe, nom et verbe étant, dans ce cas, des formes générales encore vides de matière (Joly $1984: 46)$.

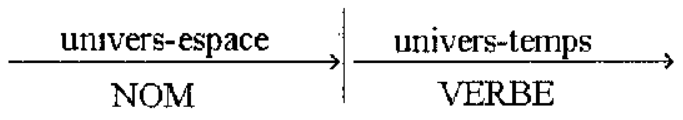

Suivant le mécanisme d'incidence, c'est-à-dire le type de rapport de l'apport de signification au support de signification, on peut distinguer dans chaque plan trois niveaux de réalisations (ibid. : 46-48). Au niveau J, immédiatement sous les formes aperturales du nom et du verbe, on trouve le substantif (= nom-substantif) et l'infinitif (= verbe-substantif) ; au niveau II, d'un côté l'adjectif (= nom adjectif), de l'autre les participes et toutes les formes du verbe conjugué en personnes; au niveau III, dans le plan du nom, l'adverbe d'adjectif (adjectif d'adjectivation), dans le plan du verbe, l'adverbe de verbe.

\begin{tabular}{|l|l|l|}
\hline & \multicolumn{1}{|c|}{$\begin{array}{c}\text { PLAN DU NOM } \\
\text { (univers-espace) }\end{array}$} & \multicolumn{1}{c|}{$\begin{array}{c}\text { PLAN DU VERBE } \\
\text { (univers-temps) }\end{array}$} \\
\hline niveau I & substantif & infinitif \\
\hline niveau II & adjectif' & participe verbe conjugué \\
\hline niveau III & adverbe d'adjectif & adverbe de verbe \\
\hline
\end{tabular}


Pour l'infinitif, il représente un cas d'incidence interne correspondant en gros à celui du substantif. L'apport de signification n'est effectivement incident à aucun support en dehors de ce que signifie l'infinitif. L'apport de signification ne diffère donc pas du support de signification. En revanche. les autres modes nominaux (les participes) ainsi que le verbe conjugué sont incidents à un support de signification extérieur à eux-mêmes. On dira un homme courant, un animal courant. Le participe courant, dont l'incidence est externe, est habile à se dire de toutes sortes de supports. Il n'en est pas de même de l'infinitif courir, qui ne peut se dire que de ce qu'il signifie en soi :

\section{Courir me fatigue.}

Par contre, le verbe conjugué est incident à la personne, nominale ou pronominale.

L'apport de signification de l'adjectif a aussi son incidence à un support de signification extérieur à ce qu'il désigne. Ce support est un substantif. L'incidence de l'adjectif est donc aussi externe. Quant à l'apport de signification de l'adverbe, il est incident, non pas exactement au support de signification de l'adjectif, mais à l'incidence de l'adjectif au substantif. Nous sommes ici en présence d'une incidence à une incidence. Comme celle de l'adjectif, l'incidence de l'adverbe est externe, mais de second degré.

D'après Guillaume, l'infinitif qui relève en tant que tel du plan du verbe, présente des capacités fonctionnelles, conformément au schéma ci-dessus, non pas du verbe, mais celles du nom. Il prend position dans le plan même du verbe à proximité extrême du plan du nom. En d'autres termes, l'infinitif est dans le plan du verbe un mot qui participe de la nature du nom, autant que cela est possible sans quitter le plan du verbe.

Les observations présentées ci-dessus montrent non seulement l'infinitif comme catégorie intermédiaire entre le verbe et le nom, mais aussi elles mettent en évidence le caractère hétérogène du seul concept de catégorie grammaticale. Ainsi, comme nous l'avons vu plus haut, la catégorie du verbe comporte des sous-classes qui diffèrent par le nombre de propriétés verbales et nominales. Si nous ajoutons à notre liste le participe, par exemple sachant, et que nous le comparions à l'infinitif savoir et à la forme sachez, nous verrons, entre autres, que seule la forme de l'impératif est apte à exprimer la catégorie de la personne, et que seul l'infinitif a la faculté d'assumer la fonction de prime actant dans la phrase, ce qui le rapproche du nom. Nous avons pourtant remarqué que certains indices classificateurs qui traditionnellement permettent de définir.une catégorie ne font toujours pas preuve. Et même si nous réservons, d'après Guillaume, l'univers temps à la catégorie du verbe, nous trouverons des éléments relevant de l'univers espace (noms, adjectifs) aptes à exprimer certaines valeurs temporelles.

Enfin, nous avons vu que pour que l'étude de l'opposition entre catégories grammaticales soit exhaustive, il est indispenseble de l'étendre à d'autres langues qui présentent des schémas d'organisation de la phrase différents, même si ces 
langues sont parlées par des communautés relativement peu nombreuses. Car il est évident que les conclusions concemant des concepts aussi généraux et universels que celui de catégorie grammaticale, ne seront complètes et convainquantes qu'après avoir pris en compte tous les systèmes langagiers du monde.

\section{BIBLIOGRAPHIE}

Cha ra ud ea u P. (1992), Grammaire du sens et de l'expression, Paris.

Guill a ume G. (1973), Langage et science du langage, Paris-Québec.

Guilla ume G. (1987), Leçons de linguistique, 1945-1946, série A, vol. 7, Villeneuve-d'Ascq (Nord).

Joly A. (1984), La distinction du nom et du verbe dans la théorie de G. Guillaume, Modèles linguistiques, t. Vl, fasc. 1, pp. 41-52.

Lazard G. (1984), La distinction entre nom et verbe en morphologie et en syntaxe, Modèles linguistiques, $t$. VI, fasc. 1, pp. 29-39.

Martinet A. (1965), La linguistique synchronique. Études et recherches, Paris.

Le Nouveau Petit Robert. Dictionnaire alphabétique et analogique de la langue française (1993), réd. dirigée par Rey Debove J. et Rey A.. Paris.

Tesnièr e L. (1965), Éléments de syntaxe structurale, Paris. 